\title{
DUST IN THE LARGE MAGELLANIC CLOUD
}

HUGH M. JOHNSON*

Lockheed Missiles and Space Company, Palo Alto, Calif., U.S.A.

\begin{abstract}
A brief review of extinction effects is followed by a discussion of new Cerro Tololo Schmidt plates. They were exposed on parts of the LMC with filters which reveal the presence or absence of a continuum, such as one scattered on dust, in $\mathrm{H}$ il regions. The results are presented in comparison with the Orion nebula which was observed in the same way. The continuum is always relatively weak in those LMC H II regions which are significantly larger than Orion, but the continuum averages strong in the Orion-class and point-image nebulae.
\end{abstract}

\section{Extinction Effects}

Shapley (1951) made counts of extragalactic nebulae through the LMC and derived extinction of the order of nil to $0.27 \mathrm{mag}$. in several areas. From UBV photometry the maximum extinction affecting any part of the LMC in open regions is $A_{V}=0.5$ mag. and $0.2-0.3 \mathrm{mag}$. on the average; in young associations, especially those embedded in gaseous nebulosity, the average rises to $A_{V}=0.4-0.5 \mathrm{mag}$. outside $30 \mathrm{Dor}$, and 1.3 mag. in 30 Dor (Thackeray, 1963; Bok, 1966). Visvanathan (1966) measured the polarization of 30 stars in the LMC and found values up to 0.068 mag. Brück et al. (1970) went on to conclude that the optical properties of dust grains in the Magellanic Clouds resemble those in the anticenter (Perseus) region of the Galaxy (in contrast with the Cygnus region.) Lastly, Hodge (1972) has catalogued 68 dark nebulae in the LMC $1^{\prime}-21^{\prime}$ in size. The survey he made was incomplete where silhouetting was weak or confused by $\mathrm{H}$ II regions.

\section{Bright Continua}

We expect that scattering on dust would produce reflection nebulae in the LMC, or that atomic continua or nonthermal synchrotron emission might be found (Johnson, 1971). Although emission nebulae are abundant in the LMC, it is not clear that reflection nebulae have been reported. Most photometric effort (Doherty et al., 1956; Feast, 1964; and Dickel, 1965) has gone into measuring atomic-line intensities. Henize (1956) notes several spectral continua in emission nebulae of the LMC, but he remarks that "in general the presence of a continuum probably indicates the existence of a cluster or group of stars whose extent is about equal to that of the nebulosity."

\footnotetext{
* Visiting Astronomer, Cerro Tololo Inter-American Observatory, which is operated by the Association of Universities for Research in Astronomy, Inc., under contract with the National Science Foundation.
} 
TABLE I

Curtis Schmidt plates of the LMC and Orion

\begin{tabular}{lcllc}
\hline 1970 January (UT) & CTIO plate No. & $\begin{array}{l}\text { Field center } \\
\text { RA (1950) Dec. }\end{array}$ & Passband & Exposure (min) \\
\hline 10 & 5777 & $05^{\mathrm{h}} 35^{\mathrm{m}} \cdot 0-67^{\circ} 29^{\prime}$ & $N$ & 60 \\
10 & 78 & $05^{\mathrm{h}} 35^{\mathrm{m}} \cdot 0-67^{\circ} 29^{\prime}$ & $W$ & 3 \\
11 & 85 & $0533.9-0524$ & $N$ & 120 \\
11 & 86 & $0533.9-0524$ & $W$ & 5 \\
11 & 87 & $0538.4-6958$ & $W$ & 3.6 \\
11 & 88 & $0538.4-6958$ & $N$ & 35.3 \\
12 & 90 & $0505.2-6807$ & $N$ & 120 \\
12 & 91 & $0505.2-6807$ & $W$ & 5 \\
13 & 93 & $0501.6-7034$ & $N$ & 120 \\
13 & 94 & $0501.6-7034$ & $W$ & 5
\end{tabular}

\section{New Observations}

We have taken plates of parts of the LMC and comparison plates of the Orion nebula with the Curtis Schmidt telescope of the Cerro Tololo Inter-American Observatory, as listed in Table I. All plates were exposed in a clear and moonless sky on filtered IIa-O emulsion. The ' $N$ ' filter restricted field size to $3.8 \mathrm{sq}$, and the ' $W$ ' filter to $4.1 \mathrm{sq}$. In both passbands the LMC fields overlap and cover a total area of about $38 \mathrm{sq} \mathrm{deg.}$

\section{The Wide and Narrow Passbands}

The wide $(W)$ band is bounded at the upper wavelength limit by the sensitivity of the emulsion, and at the lower limit by a Wratten 2B filter, giving 50 per cent of peak transmission near $\lambda 4100$ and $\lambda 5000$. The narrow $(N)$ band is produced by a BairdAtomic interference filter designed to peak at $\lambda 4690 \pm 5$ in the $f / 3.5$ beam of the camera. FWHM $=70 \AA \pm 5 \AA$, and transmission is less than $3 \%$ in the band wings below $\lambda 4610$ and above $\lambda 4780$. The measurements of the interference filter have been made in the spectrophotometer of the Kitt Peak National Observatory* with the aid of J. C. Golson, and in the spectrophotometer of the Hale Observatories by W. C. Miller. It has been checked to be uniform in its characteristics within a few per cent in a sample of different areas and to be stable over a period of more than a year. There are no leaks outside the specified passband in the range of sensitivity of IIa-O emulsion. The spectrophotometer data were the basis for an extrapolation to a more convergent beam on the theory of Lissberger and Wilcock (1959).

Exposures in the two passbands with the ratios of Table I (except plates 5787-88) nearly equalize the images of most stars. The inverse exposure-ratio exceeds FWHM $(W$-band $) / \operatorname{FWHM}(N$-band $)=900 \AA / 70 \AA$ by a factor of about 2 simply because of reciprocity failure of the emulsion. Near-equality of effective wavelengths practically

* The Kitt Peak National Observatory is operated by the Assocation of Universities for Research in Astronomy, Inc., under contract with the National Science Foundation. 
eliminates effects of interstellar reddening. Stars such as Wolf-Rayet stars and highexcitation nebulae with $\mathrm{He}$ II $\lambda .4686$ emission have unequal images, stronger in the $N$ band, as Reddish (1968) first demonstrated with a similar pair of passbands. However, low-excitation nebulae have no He II $\lambda 4686$ emission, and their line spectrum contributes little to the $N$ band. Orion and the LMC nebulae which have been classified (Dickel et al., 1964) are all of fairly low and nearly equal excitation class (3-4).

TABLE II

Schmidt telescope response to three compositions of light

Category Composition

Predicted ratio of total light in $N$ band to total light in $W$ band

$\begin{array}{lll}\text { (a) } & \text { Low-excitation lines (Orion less continuum) } & 0.005 \\ \text { (b) } & \text { Orion nebula (continuum plus low-excitation lines) } & 0.5 \\ \text { (c) } & \text { Pure continuum } & 1.0\end{array}$

Table II compares three categories of interest for the study of the LMC. For the Orion nebula we have estimated a mean intensity ratio of $\lambda .4690$ continuum to $\mathrm{H} \beta$ from O'Dell and Hubbard's (1965) data, and we have summed the intensities of lines relative to $\mathrm{H} \beta$ as reported in Johnson (1968), each line weighted by filter transmissivity. About $40 \%$ of the light in the $W$ band and $80 \%$ of the light in the $N$ band is continuum. Structural details of the Orion nebula are remarkably similar in the $N$ and $W$ images, but the $W$ image is appreciably denser, as predicted from the material compiled for Table II. We see that it should be fairly easy to distinguish categories (a), (b), and (c) of Table II among nebulae in the LMC. The Orion-nebula $N$ and $W$ images would be about $20^{\prime \prime}$ in diameter if that nebula were placed in the LMC, so it is resolved.

\section{Conclusions}

We have inspected the plates directly, and by blinking them in the Lick Observatory machine, and by superposing positive-copy films of the $W$ plates on the $N$ plates. The blink machine was made available bycourtesy of R. P. Kraft, and the film copies were made by M. M. Hanna in the photographic laboratory of the Kitt Peak National Observatory. Not all plate areas received equal attention, so this is not a homogeneous survey but rather a partial sampling of Henize (1956) emission nebulae. The sensitivity of the present combination of exposures and passbands to emission nebulae is not so great as Henize achieved with a narrow band around $\mathrm{H} \alpha$. Table III identifies the inspected nebulae and gives the dimensions and relative intensity of $\mathrm{H} \alpha$ estimated by Henize on a scale of $1-5(T=$ trace $<1)$. Point images on Henize's plates have no tabulated dimensions. The $\mathrm{H} \alpha$ intensity becomes a surface-brightness estimate for extended nebulae. The new-data column labeled $N / W$ indicates the subjective impression of the relative strength of the $N$ and $W$ images on a scale of $0-10$, with the following meaning in terms of Table-II categories: (a) should correspond to $N / W=0$ and (c) 
TABLE III

LMC nebulae examined for blue continuum

\begin{tabular}{|c|c|c|c|c|c|c|c|}
\hline Henize- $N$ & Diam.(') & $\mathrm{H} \alpha$ & $N / W$ & Henize- $\Lambda$ & Diam.(') & $\mathbf{H} \alpha$ & $N / W$ \\
\hline $4 \mathrm{~A}$ & $53 \times 50$ & 5 & 2 & $34 \mathrm{C}$ & & 2 & 10 \\
\hline 8 & $85 \times 116$ & 5 & 0 & 38 & $64 \times 52$ & 5 & 0 \\
\hline $11 \mathrm{~B}$ & $262 \times 212$ & 5 & 0 & 44B & $67 \times 57$ & 5 & 2 \\
\hline $11 \mathrm{C}$ & $191 \times 158$ & 5 & 0 & $44 C$ & $47 \times 67$ & 5 & 2 \\
\hline $11 \mathrm{E}$ & $119 \times 127$ & 4 & 0 & $44 \mathrm{D}$ & $91 \times 74$ & 5 & 1 \\
\hline $11 \mathrm{~F}$ & $199 \times 97$ & 4 & 0 & 49 & $68 \times 82$ & 5 & 3 \\
\hline 16 & & 3 & 6 & 59 & $152 \times 174$ & 4 & 1 \\
\hline 18 & & 4 & 6 & $59 \mathrm{~B}$ & $117 \times 118$ & 4 & 0 \\
\hline 19 & & $\mathbf{T}$ & 9 & $63 \mathrm{~A}$ & $40 \times 40$ & 5 & 3 \\
\hline 22 & & 5 & 9 & $77 \mathrm{~A}$ & $30 \times 32$ & 5 & 2 \\
\hline $23 \mathrm{~A}$ & $82 \times 107$ & 3 & 1 & $79 \mathrm{~A}$ & $78 \times 75$ & 5 & 2 \\
\hline 24 & & 2 & 10 & $79 B$ & $21 \times 20$ & 5 & 8 \\
\hline 25 & & 2 & 10 & 79C, D, & $180 \times 154$ & 3 & 2 \\
\hline $26^{a}$ & $27 \times 30$ & 4 & 10 & 82 & & 5 & 10 \\
\hline $27^{\mathrm{a}}$ & $48 \times 53$ & 3 & 10 & 83 & $340 \times 297$ & 3 & 1 \\
\hline 28 & & 2 & 10 & 85 & $23 \times 23$ & 2 & 6 \\
\hline 29 & & 2 & 10 & 87 & $19 \times 14$ & 4 & 10 \\
\hline $30 \mathrm{~A}$ & $30 \times 41$ & 4 & 0 & 88 & $36 \times 21$ & 2 & 10 \\
\hline $30 \mathrm{~B}$ & $25 \times 17$ & 3 & 6 & 89 & & 4 & 3 \\
\hline 32 & $16 \times 19$ & 2 & 10 & $90^{\mathrm{a}}$ & $25 \times 27$ & 4 & 10 \\
\hline 33 & $81 \times 69$ & 4 & 0 & $91 \mathrm{~A}, \mathrm{~B}$ & $32 \times 48$ & $4-5$ & 2 \\
\hline $34 \mathrm{~A}$ & & 3 & 10 & $93^{a}$ & $41 \times 26$ & 1 & 10 \\
\hline 34B & & 2 & 10 & $95^{\mathrm{a}}$ & $16 \times 13$ & 1 & 9 \\
\hline 96 & & 2 & 10 & 138 & $391 \times 363$ & 1 & 0 \\
\hline 97 & & 3 & 9 & $157 \mathrm{~A}$ & $922 \times 1063$ & 5 & 2 \\
\hline 98 & & 2 & 10 & 182 & & 3 & 2 \\
\hline 99 & & 1 & 10 & 183 & & 2 & 10 \\
\hline 101 & & 1 & 10 & $186 A$ & & 3 & 5 \\
\hline 102 & & 2 & 10 & 186B & $38 \times 36$ & 3 & 10 \\
\hline $105 \mathrm{~A}$ & $160 \times 270$ & 4 & 2 & $186 C^{a}$ & $27 \times 28$ & 2 & 10 \\
\hline 106 & & 1 & 10 & 187 & & 1 & 10 \\
\hline $109^{a}$ & $24 \times 29$ & 1 & 10 & 188 & & 2 & 10 \\
\hline 111 & & 1 & 10 & 190 & $114 \times 133$ & 3 & 2 \\
\hline 112 & $48 \times 38$ & 4 & 2 & 192 & & 2 & 10 \\
\hline $113 \mathrm{~A}$ & $21 \times 29$ & 5 & 1 & $193 \mathrm{~A}$ & $29 \times 25$ & 5 & 6 \\
\hline $113 C$ & $81 \times 65$ & 4 & 1 & $193 B^{a}$ & $32 \times 36$ & 2 & 10 \\
\hline 113D & $65 \times 73$ & 4 & 1 & $193 C^{a}$ & $38 \times 52$ & 2 & 10 \\
\hline $113 \mathrm{~F}$ & $49 \times 44$ & 4 & 1 & $193 D^{a}$ & $29 \times 35$ & $T$ & 10 \\
\hline 115 & $38 \times 37$ & 1 & 10 & $193 E^{a}$ & $29 \times 35$ & $T$ & 10 \\
\hline 117 & $28 \times 27$ & 4 & 10 & $194^{a}$ & $16 \times 19$ & 3 & 8 \\
\hline 118 & $26 \times 27$ & 4 & 5 & $195 \mathrm{~A}$ & $34 \times 27$ & 4 & 6 \\
\hline 119 & $800 \times 932$ & 3 & 1 & 195B & $46 \times 32$ & 4 & 1 \\
\hline 120 & $520 \times 365$ & 3 & 1 & & & & \\
\hline
\end{tabular}

a $N$ and $W$ images appear to be stellar.

to $N / W=10$ (equal intensities). The plates of Orion (b) are estimated at $N / W=7$. Thus the subjective scale is no doubt related nonlinearly to real intensity ratios and is multiplied by 10 . 
An interesting difficulty is that 12 non-stellar $\mathrm{H} \alpha$ images of the Henize (1956) catalog appeared to be stellar in $N$ and $W$. These are superscripted ' $\mathrm{a}$ ' in Table III. Only three of them are noted by Henize with any continuum around $\mathrm{H} \alpha$. Although the small scale of Henize's finding charts admit the possibility of misidentifications, most of the 12 images which are discrepant cannot be so explained. It is possible then that a blue stellar continuum, or a concentrated nebular continuum, sometimes leads to $N / W=$ 10 while the weaker, extended nebulosity around the star or central concentration is absent because of underexposure. The estimate $N / W=0$ may also result from underexposure of the $N$ image.

Disregarding the 12 asterisked observations, we find that all nebulae with point images show $N / W \geqslant 2$, and they prefer the value $N / W=10$. No definite case of $N / W>10$ was found among Henize's nebulae, but the presence of He II $\lambda .4686$ cannot be distinguished from strong continuum in cases of high $N / W$ values.

Among 'Orion-class' nebulae, i.e. resolved with geometrical mean diameters in the range $20^{\prime \prime}-50^{\prime \prime}, N / W$ takes on all values in the range $0-10$. The conclusion is that nebulae with a ratio of continuum to monochromatic-line emissions like Orion's, and variants, are present in the LMC.

Among large nebuale with geometrical mean diameters $>50^{\prime \prime}, N / W \leqslant 2$. These nebulae therefore exhibit a relatively weak continuum. It is found from the data of Feast (1964), Dickel et al. (1964), and Dickel (1965) that they have rms electron densities much less than that of Orion. However, within Orion O'Dell and Hubbard (1965) determined that the ratio of continuum to monochromatic line emission at $\mathrm{H} \beta$ grew as the borders of the nebula were approached, so it may not be simply said that relatively less scattering takes place where the electron density is low.

These conclusions about the continua of the LMC nebulae may be translated into the dust/gas density ratio in so far as the scattering of starlight on dust indeed accounts for the continua in the nebulae. The degree to which dust is responsible for continua in emission nebulae is a complicated subject in itself, and atomic continua are often taken to be dominant (e.g. Pottasch, 1965).

Three nebulae in Table III are of special interest: N 49, N 63A, and N 157A (30 Dor), which are among four LMC objects known to have a nonthermal radiofrequency spectrum (Mathewson and Healy, 1964). But their optical behavior in $N / W$ is not notably different from the other nebulae observed in this work. The $N$-image details of 30 Dor imitate the $W$-image details closely, as in the images of Orion, but $N / W$ is considerably weaker than it is in Orion. The fourth nonthermal nebula, N 132D, is in a very dense field of stars, which prevents satisfactory examination.

\section{Acknowledgement}

This work has been done under the Lockheed Independent Research Program. 


\section{References}

Bok, B. J.: 1966, Ann. Rev. Astron. Astrophys. 4, 95.

Brück, M. T., Lawrence, L. C., Nandy, K. N., Thackeray, A. D., and Wood, R.: 1970, Nature 225, 532.

Dickel, H. R.: 1965, Astrophys. J. 141, 1306.

Dickel, H. R., Aller, L. H., and Faulkner, D. J.: 1964, in F. J. Kerr and A. W. Rodgers (eds.), The Galaxy and the Magellanic Clouds, Australian Acad. Sci., Canberra, p. 294.

Doherty, L., Henize, K. G., and Aller, L. H.: 1956, Astrophys. J. Suppl. $2,345$.

Henize, K. G.: 1956, Astrophys. J. Suppl. 2, 315.

Hodge, P. W.: 1972, Publ. Astron. Soc. Pacific 84, 365.

Johnson, H. M.: 1971, in A. B. Muller (ed.), The Magellanic Clouds, D. Reidel Publ. Co., DordrechtHolland, p. 95.

Johnson, H. M.: 1968, in B. M. Middlehurst and L. H. Aller (eds.), Nebulae and Interstellar Matter, University of Chicago Press, Chicago, p. 63.

Lissberger, P. H. and Wilcock, W. L.: 1959, J. Opt. Soc. Am. 49, 126.

Mathewson, D. S. and Healey, J. R.: 1964, in F. J. Kerr and A. W. Rodgers (eds.), The Galaxy and the Magellanic Clouds, Australian Acad. Sci., Canberra, p. 283.

O'Dell, C. R. and Hubbard, W. B.: 1965, Astrophys. J. 142, 591.

Pottasch, S. R.: Vistas in Astronomy 6, 149.

Reddish, V. C.: 1968, Observatory 88, 139.

Shapley, H.: 1951, Proc. Nat. Acad. Sci. 37, 136.

Thackeray, A. D.: 1963, Adv. Astron. Astrophys. 2, 264.

Visvanathan, N.: 1966, Monthly Notices Roy. Astron. Soc. 132, 423. 